\title{
Remission death in acute lymphoblastic leukaemia: a changing pattern
}

\author{
A Atra, S M Richards, J M Chessells
}

\begin{abstract}
The pattern of remission deaths was examined in 842 children with acute lymphoblastic leukaemia (ALL) treated , at a single centre over 18 years. The mortality rate from leukaemia fell significantly during three consecutive time periods during which treatment became progressively more intensive and that during remission induction fell from $3 \cdot 5 \%$ to under $1 \%$, but the rate of death in remission stayed constant at $5-6 \%$.

The factors associated with an increased risk of remission death were: young age, a higher leucocyte count, bone marrow transplantation, and Down's syndrome. The pattern of remission deaths changed over the years; measles and herpes viruses decreased while deaths associated with periods of intensification and gut toxicity increased. Four children developed second neoplasms.

Treatment of ALL is still associated with a significant risk of death in remission but the pattern of infective deaths has changed. Many should be avoidable by provision of adequate supportive care, close supervision after periods of intensive treatment, and appropriate antibiotic, antifungal, and cytokine therapy. (Arch Dis Child 1993; 69: 550-554)
\end{abstract}

The treatment of acute lymphoblastic leukaemia (ALL) in childhood has become progressively both more successful and more intensive and thus possibly associated with more short and long term toxicity. ${ }^{1}$ Induction and intensification treatment are associated with myelosuppression and gastrointestinal toxicity, both predisposing to bacterial and sometimes fungal infections, while both cranial irradiation and continuing (maintenance) treatment are immunosuppressive, ${ }^{23}$ thus predisposing to non-bacterial infections. We have reviewed the causes of death in remission in three consecutive cohorts of children treated at the Hospitals for Sick Children to determine whether the pattern of such deaths had changed with increasing intensity of treatment over the years and to identify those patients who might be deemed at special risk of death in remission.

\section{Patients and methods}

The patients comprise all of the children referred to the Hospitals for Sick Children for treatment of ALL from 1972 to 1989 inclusive. Most of the patients in the first cohort were the subject of a previous paper. ${ }^{4}$

All children received induction treatment with prednisolone, vincristine and colaspase, frequently with additional drugs, all received central nervous system directed treatment with intrathecal methotrexate and cranial irradiation, deferred in those under 2 and omitted in those under 1 year from 1985 onwards and all received continuing (maintenance) chemotherapy for at least two years. The continuing treatment comprised 6mercaptopurine and methotrexate with prednisolone and vincristine and, in the earlier protocols, some additional drugs.

The protocols for treatment had become progressively more intensive over the years but the patients could conveniently be assigned to one of three groups according to the years of diagnosis.

From 1972-8 (group 1) patients received early MRC UKALL protocols II-IV which have been reviewed in detail elsewhere. ${ }^{5}$ From 1979-82 (group 2) children with B-precursor ALL received our PLOD protocol described in detail previously, ${ }^{6}$ while those with $\mathrm{T}$ cell ALL were treated on a multidrug combination chemotherapy schedule devised by the UK Children's Cancer Study Group. ${ }^{7}$ From 1983 (group 3) onwards children were treated on the pilot protocol for MRC UKALL X, or the definitive trial, ${ }^{8}$ the main additional feature of which is five day blocks of intravenous cytotoxic treatment given at five and/or 20 weeks from díagnosis, or neither. The features and toxicity of these intensification blocks have been described. ${ }^{9}$

From 1980 onwards, a small number of children deemed at high risk of treatment failure, as determined by an initial leucocyte count in excess of $100 \times 10^{9} / 1$ have been treated by chemotherapy, total body irradiation (or chemotherapy alone in the case of infants), and bone marrow transplantation from a histocompatible sibling donor.

All patients have been followed up until the end of 1992, a minimum of one year off treatment. We included patients diagnosed up to the end of 1989 to include the last year 
Table 1 Remission deaths during three time periods

\begin{tabular}{llllllll}
\hline Years & Group & $\begin{array}{l}\text { Total } \\
\text { No }\end{array}$ & $\begin{array}{l}\text { All } \\
\text { deaths }\end{array}$ & O/E & Remitting & $\begin{array}{l}\text { Remission } \\
\text { deaths }\end{array}$ & O/E \\
\hline $1972-78$ & 1 & 298 & 161 & $1 \cdot 39$ & 287 & 18 & $1 \cdot 15$ \\
$1979-82$ & 2 & 214 & 88 & $1 \cdot 01$ & 210 & 10 & 0.88 \\
$1983-89$ & 3 & 330 & 81 & 0.64 & 328 & 17 & 0.95 \\
Totals & & 842 & 330 & - & 825 & 45 & - \\
\hline
\end{tabular}

$\mathrm{O} / \mathrm{E}=$ observed/expected . $(\mathrm{p}<0.00001)$. The early death rate is now less than $1 \%$ (two in 330 cases). There has, however, been no parallel fall in the rate of death in remission which remains between $5 \%$ and $6 \%$.

RISK FACTORS FOR DEATH IN REMISSION

Table 2 shows the results of analysis of the risk of remission death in the 825 children who achieved remission. There was no apparent effect of gender, but younger children, particularly under 2 years and older children were at greater risk than those between 2 and 10 years of age. Patients with Down's syndrome were vulnerable and, not surprisingly, the risk of remission death in children with a high leucocyte count (who tended to receive more intensive treatment) and those receiving bone marrow transplantation is greater than others.

\section{Results}

The number of children in each group is shown in table 1 . A total of 842 children started treatment of whom 825 (98\%) achieved remission. Remission was not achieved in 17 children. There were 11 deaths without remission in group 1, five from Gram negative sepsis as previously described, ${ }^{11}$ two from complications of a high leucocyte count and hyperviscosity (leucostasis) and haemorrhage, and four who failed to respond to chemotherapy. Four children had early deaths in group 2, one from hepatic failure and sepsis, and two from leucostasis and bleeding, while one child failed to respond to chemotherapy. One child in group 3 died from Escherichia coli septicaemia during induction and one from leucostasis.

The overall survival rate, as shown in table 1, has improved steadily during this time

Table 2 Risk factors for death in remission $\star$

\begin{tabular}{|c|c|c|c|c|}
\hline Factors & Total & Observed & $O / E$ & $p$ Value \\
\hline \multicolumn{5}{|l|}{ Sex } \\
\hline Male & 472 & 22 & $0 \cdot 87$ & NS \\
\hline & 41 & 5 & $3 \cdot 83$ & \\
\hline $1-<2$ & 76 & 8 & 1.96 & \\
\hline $2-10$ & 626 & 27 & $0 \cdot 76$ & 0.0009 \\
\hline$\geqslant 10$ & 82 & 5 & $1 \cdot 20$ & \\
\hline \multicolumn{5}{|c|}{ White cell count $\left(\times 10^{9} / 1\right)$} \\
\hline$<50$ & 634 & 31 & $0 \cdot 85$ & \\
\hline$\geqslant 50$ & 191 & 14 & 1.63 & 0.03 \\
\hline \multicolumn{5}{|c|}{ Down's syndrome } \\
\hline Yes & 12 & 3 & 4.98 & \\
\hline No & 813 & 42 & 0.95 & 0.01 \\
\hline \multicolumn{5}{|c|}{ Bone marrow transplant } \\
\hline No & $808 t$ & 42 & 0.95 & \\
\hline Yes & 15 & 3 & 3.96 & 0.02 \\
\hline
\end{tabular}

$\mathrm{O} / \mathrm{E}=$ observed/expected.

${ }^{\star} \mathrm{p}$ Value $=$ for heterogeneity from log rank analysis.

t $\mathrm{p}$ Value $=$ for heterogeneity from log rank analysis.
Two received autologous transplants in first remission.
THE PATTERN OF REMISSION DEATHS (TABLE 3) most common cause of death in groups 1 and 2 , all but one child had an interstitial pneumonia; the remaining girl had an encephalitis. There have been no fatal cases from 1983 onwards. The single case of fatal chickenpox occurred in group 1 and two children have died from disseminated herpes simplex infection, one in group 1 and one in group 3 . There were three cases of cytomegalovirus infection in group 1; one child dying from cytomegalovirus pneumonia during treatment, and one developing chronic respiratory failure who ment. The third child, who was blind from cytomegalovirus retinitis and who excreted cytomegalovirus for many years, eventually died 13 years from diagnosis with acute cardiac failure; postmortem examination showed evidence of myocarditis the aetiology of which was not clear. Two of these children had received granulocyte transfusions.

Two children in group 2 died from Pneumocystis carinii pneumonitis, but, after the introduction of routine prophylactic cotrimoxazole, no further fatalities have occurred.

A total of seven children died from proved bacteraemias (table 3). Four of these have been previously reported in the patients in group 1: two with Pseudomonas aeruginosa, one with Haemophilus influenzae, and one with Clostridium sp. ${ }^{4}$ There were no deaths associated with confirmed bacteraemia in group 2 , but three children died in group 3. One death
Measles, or presumed measles, was the single died within a year of electively stopping treat-

Table 3 Type of remission death

\begin{tabular}{|c|c|c|c|c|c|c|c|c|}
\hline Group & Measles & $\begin{array}{l}\text { Herpes } \\
\text { viruses }\end{array}$ & Cytomegalovirus & $\begin{array}{l}\text { Pneumocystis } \\
\text { carinii } \\
\text { pneumonia }\end{array}$ & Bacteraemia & $\begin{array}{l}\text { Other } \\
\text { infections }\end{array}$ & Non-infective & Total \\
\hline $\begin{array}{l}1 \\
2\end{array}$ & $\begin{array}{l}6 \\
3\end{array}$ & $\begin{array}{l}2 \\
0\end{array}$ & $\begin{array}{l}3 \\
0\end{array}$ & $\begin{array}{l}0 \\
2\end{array}$ & $\begin{array}{l}4 \\
0\end{array}$ & 1 & $\begin{array}{l}\text { Suicide (1), myelodysplasia (1) } \\
\text { Haemorrhage (1), Langerhans } \\
\text { cell histiocytosis (1) }\end{array}$ & $\begin{array}{l}18 \\
10\end{array}$ \\
\hline 3 & 0 & 1 & 0 & 0 & 3 & 11 & Brain tumour (1), lymphoma (1) & 17 \\
\hline Totals & 9 & 3 & 3 & 2 & 7 & 15 & 6 & 45 \\
\hline
\end{tabular}

^See table 4 for details. 
Table 4 Details of miscellaneous infections

\begin{tabular}{ll}
\hline Group 1 & Interstitial pneumonia (1) \\
Group 2 & Mycoplasma pneumonia (2) \\
& JC encephalitis (1) \\
Group 3 & Interstitial pneumonia (1) \\
& Encephalitis (2) \\
& Gastrointestinal (4) \\
& Bone marrow transplant related (3) \\
& Candidiasis (1) \\
\hline
\end{tabular}

occurred from $H$ influenzae meningitis in a boy five months off treatment, while two children had infection associated with coagulase negative staphylococci. In one of these the organism was isolated from blood cultures in a boy with an apparent haemophagocytic syndrome which persisted despite antibiotics and withdrawal of cytotoxic treatment; permission for postmortem examination was refused. The second child developed positive blood cultures in association with an endocarditis and Hickman catheter; she died after embolic episodes despite catheter removal and antibiotics.

There were a total of 15 children who died from other infections or presumed infections (table 4). Two had severe interstitial pneumonia, one in group 1 and one in group 3, and no causative organism was identified. Two children, both with Down's syndrome, died from pneumonia with serological evidence of mycoplasma infection. There were three deaths from encephalitis; one associated with mumps infection, one with JC papovavirus and one acute encephalitis in which there was no bacterial or viral isolate and permission for postmortem examination was refused. Four children in group 3, two of whom were infants under 1 year of age and one had Down's syndrome, died after intensification treatment with collapse, severe gut toxicity, and presumed sepsis.

There were three deaths after bone marrow transplantation, two, both in infants, from adenovirus pneumonitis and one from acute graft-versus-host disease. One girl in group 3 died from hepatosplenic candidiasis; the diagnosis was made on open liver biopsy and she failed to respond to prolonged treatment with intravenous amphotericin.

\section{NON-INFECTIVE DEATHS, LATE DEATHS, AND SECOND MALIGNANCY}

One boy in group 2 collapsed and died after intensive chemotherapy; sepsis was clinically suspected but he died despite intensive supportive treatment and postmortem examination showed extensive haemorrhage at the base of the brain and in the spinal cord. No organisms were isolated from any cultures.

Five children died from causes other than malignancy more than three months after treatment was electively stopped. Two of the children with cytomegalovirus described above died off treatment, one from chronic respiratory failure and one from cardiotoxicity. One boy, as described above died from $H$ influenzae meningitis. One boy committed suicide at the age of 21 years.
Four children have developed second malignancies. One had secondary myelodysplasia with monosomy 7 and one boy developed a parietal glioma. The third child, with a family history of neurofibromatosis, two of whose siblings subsequently developed mediastinal non-Hodgkin's lymphoma, presented with ALL of early pre-B subtype, and then developed a lymphoblastic lymphoma of the mediastinum.

The fourth patient, a girl with $T$ cell ALL, developed an illness with some features of haemophagocytic lymphohistiocytosis but postmortem examination confirmed a diagnosis of Langerhans cell histiocytosis.

\section{Discussion}

This analysis of all children treated at a single centre during 18 years shows, as expected, that there has been a highly significant improvement in survival for children with ALL. The early death rate remains low and is less than $1 \%$ despite increased intensity of induction treatment. However, the rate of remission deaths has not changed and remains between 5 and $6 \%$. There has, however, been a change in the pattern of remission deaths which is illustrated by our results and we have also been able to identify certain vulnerable groups of patients.

The first of these are children under 2 years, and infants in particular. Infants under the age of 1 year with ALL have an extremely high relapse rate ${ }^{1213}$ and our attempts to intensify treatment have included further high dose consolidation and bone marrow transplantation. It is significant that two recent deaths in infants occurred from gut toxicity during intensification and two from adenoviral infection after bone marrow transplantation. While attempts to improve prognosis in this high risk group of patients are clearly justified, the protocols require constant modification in the light of toxicity and availability of a high level of supportive care.

The second group are children with Down's syndrome, whose vulnerability we have previously noted. ${ }^{14}$ These children have a pre-existing immunodeficiency, ${ }^{15}$ which is presumably exacerbated by continuing chemotherapy. It is of interest that both deaths were apparently associated with mycoplasma pneumonitis.

The third group of children are those undergoing bone marrow transplantation in first remission. Bone marrow transplantation reduces the risk of relapse in a select group of high risk patients with ALL ${ }^{16}$ but is associated with a greater morbidity and mortality, thus emphasising the need for careful selection of patients at highest risk of treatment failure.

The change in protocols over the years has been accompanied by a change in the pattern of remission deaths. The classical maintenance or continuing treatment given to children with ALL is immunosuppressive rather than myelosuppressive $^{2}$ and thus increases susceptibility to non-bacterial infections. These were classically measles and herpes virus infections, 
together with $P$ carinii pneumonitis. Fortunately all of these infections have become less important as a cause of death in remission. The fall in measles infections in our population, which preceded the introduction of measles, mumps and rubella vaccine, should happily now be sustained, thus eliminating one of the most serious and untreatable infections.

No child has died with chickenpox for over 17 years thanks to the availability of acyclovir and zoster immunoglobulin. Herpes simplex is responsive to treatment with acyclovir and deaths should be preventable by liberal early use of the drug in children with severe and or recurrent oral herpes. It is of interest that two of the three deaths associated with cytomegalovirus infection occurred in children who had received several granulocyte transfusions and that there have been no deaths in recent years.

Our original approach to diagnosis of $P$ carinii pneumonitis with a strict policy of early lung biopsy was followed by reluctance to use this investigation after the introduction of cotrimoxazole for treatment. Both the deaths from pneumocystis pneumonitis occurred in children who had failed to respond to cotrimoxazole and in whom extensive investigation was pursued rather late. More recently, the routine use of prophylactic co-trimoxazole, bronchioalveolar lavage in diagnosis and steroids in selected cases, have improved prevention, diagnosis, and treatment. ${ }^{17}$

Over half the deaths in groups 1 and 2 were due to these four types of non-bacterial infection. There was a significant change in the pattern in children in group 3 where only one of the 17 deaths occurred from herpes simplex infection but there was a miscellany of other causes, many of which could be attributed to further intensification of treatment.

The introduction of further intensification treatment after children have achieved remission increases the risk of bacterial infection as during induction, and, dependent on type of protocol, there may be additional gastrointestinal toxicity. This was our original experience with the development of MRC UKALL $\mathrm{X}^{9}$ and lead to subsequent modification of the protocol. Gastrointestinal toxicity contributed to two of the deaths in infants noted above and to two other deaths, one in the unmodified UKALL $\mathrm{X}$ protocol and one in a boy with Down's syndrome. Children who receive repeated courses of antibiotic treatment for fever and neutropenia are at risk of fungal infection and one of the deaths was due to hepatosplenic candidiasis. The clinical features of this syndrome have now been widely recognised ${ }^{18}$ and we have subsequently treated two children successfully from this complication.

The pattern of bacterial infections has changed in that not all the later bacteraemic deaths were due to acute Gram negative sepsis and with increasing use of intravenous catheters has come an appreciation of the risks of Gram positive bacteraemia. ${ }^{1}$

We were disappointed that the improved survival rate in the more recent series of patients was accompanied in this way by a number of bacterial infections. However, many of these infections should be potentially treatable or avoidable, by appropriate supportive care, antibiotic and antifungal treatment and judicious use of colony stimulating factors.

Lastly, it is significant that seven of the $\mathbf{4 5}$ deaths occurred after treatment had been electively stopped, three of these among the four due to second neoplasms, at least one of which had a familial basis related to neurofibromatosis with two similarly affected siblings. The association between brain tumours and ALL is well documented ${ }^{19}$ and the role of treatment, in particular cranial irradiation, in its development, remains a topic for debate. The occurrence of a new leukaemia in one child highlights the importance of cytogenetics, cytochemistry, and immunophenotyping in all leukaemic relapses. The association between Langerhans cell histiocytosis and acute leukaemia requires further study. This association has been described in 21 cases $^{20} 21$ but in the majority Langerhans cell histiocytosis has been the initial diagnosis and it has been postulated in most cases that the leukaemia may be related to previous treatment with epipodophyllotoxins. ${ }^{22}$

In conclusion, we have shown that, despite the improved survival for children with ALL, there remains a significant risk of death associated with increasing intensity of treatment. Young children, those with Down's syndrome, and those receiving intensive treatment including bone marrow transplantation are the most vulnerable. There has been a change in the pattern of infections from the common childhood viral infections to a variety of bacterial infections and gastrointestinal toxicity has been a contributory factor in some deaths. Further modifications of protocols as occurred in MRC UKALL $X$, the judicious use of cytokines, and safer bone marrow transplantation should reduce these risks and improve survival for children receiving intensive treatment. The factors contributing to the development of second malignancies need analysis in large, national studies such as those continuing under the supervision of the Childhood Cancer Research Group.

JMC and AA are supported by the Leukaemia Research Fund. We are grateful to our colleagues in histopathology for histological review, to our microbiology colleagues and especially Dr Helen Holzel for her continued support and interest and to Dr Ian Hann for his critical comments.

1 Viscoli C, Castagnola E, Rogers D. Infections in the compromised child. Ballieres Clin Haematol 1991; 41: 511-37. 2 Rapson NT, Cornbleet MA, Chessells JM, et al. Immunosuppression and serious infections in children with acute lymphoblastic leukaemia. Br $\mathcal{F}$ Haematol 1980; with acute lymphoblastic leukaemia. Br f Haematol 1980;

Nash KA, Mohammed G, Nandapalan N, et al. $\mathrm{T}$ cell function in children with acute lymphoblastic leukaemia. Br F Haematol 1993; 83: 419-27.

4 Ninane J, Chessells JM. Serious infections during continuing treatment of acute lymphoblastic leukaemia. Arch Dis Child 1981; 56: 841-4.

5 Medical Research Council. Improvement in treatment for children with acute lymphoblastic leukaemia. Lancet 1986; i: 408-11.

6 Chessells JM, Leiper AD, Tiedemann K, Hardisty RM, Richards S. Oral methotrexate is as effective as intramuscular in continuing (maintenance) therapy of acute lymphoblastic leukaemia. Arch Dis Child 1987; 62: 172-6. Mott MG, Chessells JM, Willoughy MLN, et al. Adjuvant phoma. Br fCancer 1984; 50: 457-62. 
8 Chessells JM, Bailey CC, Richards SM. MRC UKALL X; the current national trial for acute lymphoblastic leukaemia. Leukaemia 1992; 6 (suppl 2): 157-61.

9 Pinkerton CR, Bowman A, Holtzel H, Chessells JM. Intensive consolidation chemotherapy for acute lymIntensive consolidation chemotherapy for acute lymphoblastic leukaemia

10 Peto R, Pike MC, Armitage P, et al. Design and analysis of randomized clinical trials requiring prolonged observation of each patient. II Analysis and examples. $\mathrm{Br} \mathcal{F}$ Cancer 1977; 35: 1-39.

11 Chessells JM, Leiper AD. Infection during remission induction in childhood leukaemia. Arch Dis Child 1980; 55: 118-23.

12 Reaman GH, Steinherz PG, Gaynon PS. Improved survival of infants less than 1 years of age with acute lymphoblasof infants less than 1 years of age with acute lymphoblastic leukaemia treated with intensive multiagent

3 Chessells JM. Leukaemia in the young child. Br 7 Cancer 1992; 66 (suppl 18): 554-7.

14 Levitt GA, Stiller CA, Chessells JM. Prognosis of Down's syndrome with acute leukaemia. Arch Dis Child 1990; 65: $212-6$.

15 Anneren G, Magnusson CGM, Lilja G, Nordvall SL.
Abnormal serum IgG subclass pattern in children with Down's syndrome. Arch Dis Child 1992; 67: 628-31.

16 Chessells JM, Bailey C, Wheeler K, et al. Bone marrow transplantation for high-risk childhood lymphoblastic leukaemia in first remission: experience in MRC UKALL. X. Lancet 1992; 340: 565-8.

17 Masur $H$. Prevention and treatment of pneumocystis pneumonia. N Engl f Med 1992; 327: 1853-60.

18 Haron E, Feld R, Tuffnell P, et al. Hepatic candidiasis: an increasing problem in immunocompromised patients. $\mathrm{Am}$ f Med 1987; 83: 17-26.

19 Neglia JP, Meadows AT, Robison LL, et al. Second neoplasms after acute lymphoblastic leukaemia in childhood. N Engl F Med 1991; 325: 1330-6.

20 Egeler RM, Neglia JP, Puccetti DM, et al. The association of Langerhans cell histiocytosis with malignancy. Cancer 1993; 71: 865-73.

21 Arico M, Comelli A, Bossi G, et al. Langerhans cell histiocytosis and acute leukaemia: unusual association in two cases. Med Pediatr Oncol 1993; 21: 271-3.

22 Pui C-H, Ribeiro RC, Hancock ML, et al. Acute myeloid leukemia in children treated with epipodophyllotoxins for acute lymphoblastic leukemia. $N$ Engl f Med 1991; 325: $1682-7$. 